\title{
Narkose im Rettungsdienst - mehr als nur eine erfolgreiche Intubation
}

Markus Flentje, Hendrik Fiedel, Hendrik Eismann

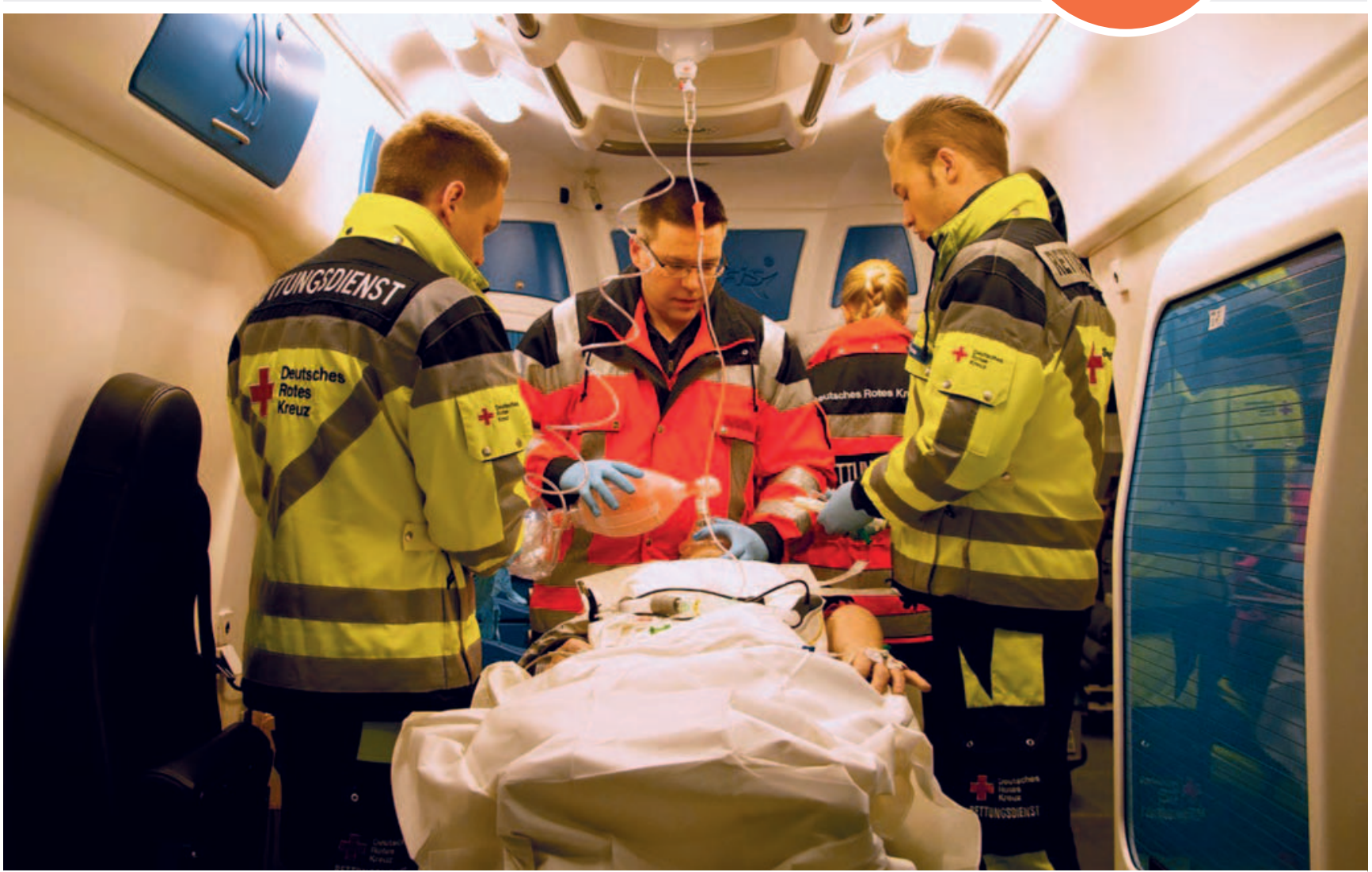

Quelle: Daniela Erhard/Thieme Gruppe

Die prähospitale Narkoseeinleitung stellt eine der kritischsten Maßnahmen in der Notfallmedizin dar. Ein instabiler Patient trifft in einer nicht optimalen Arbeitsumgebung auf ein Ad-hoc-Team, das sich am Einsatzort teilweise erstmals trifft. Die Bewältigung dieser Situation bedarf optimaler technischer Fähigkeiten jedes Teammitglieds und reibungsloser Zusammenarbeit im Team. Der Artikel beleuchtet die Teamarbeit und die pathophysiologischen Nebenwirkungen der Narkose.

\section{Rahmenbedingungen zur Narkoseeinleitung}

Die Umsetzungsvorschläge zu Medikation und Atemwegsmanagement sind in der „Leitlinie prähospitales Atemwegsmanagement" beschrieben [1,2]. Dennoch stellt die Durchführung der prähospitalen Notfallnarkose aufgrund der Rahmenbedingungen im Rettungsdienst eine besondere Herausforderung dar und gestaltet sich schwieriger als im innerklinischen Setting [3]. Einerseits wird das Ziel verfolgt, den Patientenzustand zu verbessern oder zu stabilisieren, anderseits dürfen die eingriffsbedingten Risiken und Nebenwirkungen nicht zu einer Verschlechterung der Situation des Patienten führen. Die Invasivität der medizinischen Maßnahme bedarf einer sorgfältigen Indikationsstellung [1].

\section{Indikation}

Die Narkose mit endgültiger Atemwegssicherung hat zum Ziel, den Gasaustausch eines Patienten aufrechtzuerhal- 
ten, Atemarbeit zu reduzieren und/oder eine Aspiration zu vermeiden. Als Indikation sind bewusstlose, aspirationsgefährdete, kardiopulmonal instabile und schwerverletzte Patienten vorstellbar [1]. Eine klar an spezifischen Vitalwerten orientierte Indikation gibt es selten - dies ist im Kontext eines Behandlungsverlaufs und der Bewertung einer Gesamtsituation auch schwer möglich. Einzig die Leitlinie zur Versorgung von Schädel-Hirn-Traumata gibt bezüglich der Indikation zur Narkoseeinleitung einen klaren Wert von einem GCS $<9$ vor [4].

Vor der Narkoseeinleitung ist stets zu überprüfen, ob sich der Zustand des Patienten auch ohne Narkose rasch verbessern könnte (z.B. Hypoglykämie mit tiefer Bewusstlosigkeit, da eine Glukosegabe schnell eine Zustandsverbesserung herbeiführen kann).

\section{Aspirationsgefahr}

Alle Notfallpatienten gelten als nicht nüchtern und sind damit prinzipiell stark aspirationsgefährdet. Auch bei Einhalten der Nüchternheitsgrenzen führen Stress und Schmerz zur erhöhten Produktion von Magensäure und verminderter Darmmotilität. Aus diesen Gründen unterscheiden sich die Narkoseform und der Ablauf von den häufig im Rahmen der klinischen Ausbildung erlebten innerklinischen elektiven Narkoseeinleitungen - vor allem in Ablauf der Medikamentenapplikation und der Zwischenbeatmung zur Oxygenierung.

\section{Merke}

Die Indikation einer prähospitalen Notfallnarkose muss vor dem Hintergrund von patienten-, einsatz- und anwenderbezogenen Einflüssen kritisch überprüft werden [1].

\section{Monitoring}

Um den Zustand des Patienten beurteilen zu können, ist ein möglichst umfassendes Monitoring zu etablieren. Alle angewendeten Messsysteme haben Stärken und Schwächen in ihrer Anwendung. Das EKG ist leicht ableitbar, kann aber zum kardialen Output nur Anhalte liefern. Die Pulsoxymetrie zeigt eine periphere Perfusion und Oxygenierung und ist hinsichtlich Temperatur und Verrutschen des Messsensors empfindlich. Zur Blutdruckmessung empfiehlt es sich, oszillometrische Messsysteme mit periodischen Messzyklen einzusetzen. Die Etablierung der Kapnografie zur Kontrolle des Atemwegs, Beatmungseinstellung und Beurteilung der Perfusion der Lunge ist obligat. Alle erhobenen Messwerte dürfen niemals einzeln betrachtet werden und müssen kontinuierlich von fachkundigem Personal interpretiert werden.

\section{Narkosetiefensteuerung}

Alle Medikamentengaben müssen in Dosis und Beurteilung des Wirkeintritts an den Patienten (z. B. Köperge- wicht) und seine pathophysiologische Situation angepasst werden:

- Verlängerte Kreislaufzeit (z. B. beim Schock) kann zu einem späteren Wirkeintritt der Narkose führen und triggert eine verfrühte Nachinjektion.

- Zu tiefe Narkose bewirkt eine weitere kreislaufdepressive Wirkung (innerklinische Studien zeigen nur teilweise einen Zusammenhang zwischen zu tiefer Narkose und postoperativem Tod [5, 6]).

- Bei Unterdosierung der Medikation droht eine zu flache Narkose mit möglichen Wachheitszuständen und Bronchospasmus des Patienten; diesem kann im Kreislaufschock die Möglichkeit fehlen, mit vegetativen Reaktionen zu reagieren.

- Unterschiedliche Halbwertszeiten der Medikamente sind zu beachten: Wird z. B. ein lang wirksames Muskelrelaxans eingesetzt, überschreitet es in seiner Wirksamkeit die sedierende Medikation.

\section{Nebenwirkungen und Komplikationen der Narkoseführung}

Das Behandlungsteam muss detailliertes Wissen über Wirkzusammenhänge einer Narkoseeinleitung haben, um deren Folgen im Rahmen des Situationsbewusstseins vorauszuahnen.

\section{Merke}

Bei der Narkoseeinleitung empfiehlt es sich, Katecholamine oder andere Vasopressoren vorrausschauend im Rahmen der Einleitung zu verabreichen, um Nebenwirkungen der Medikamente zu antizipieren und zu reduzieren.

\section{Hirnperfusion}

Das Gehirn stellt das zentrale Organ unseres Körpers mit hohem Sauerstoffbedarf dar, dessen Funktion es zu erhalten gilt. Der zerebrale Perfusionsdruck ergibt sich aus dem mittleren arteriellen Druck (MAD) minus des intrazerebralen Drucks (ICP). Im gesunden Gehirn ist eine humorale Autoregulation der Perfusion in den Grenzen von $60-150 \mathrm{mmHg}$ möglich [7]. Eine Hypoxie durch eine iatrogen verursachte Hypotonie kann in der Folge einen Sekundärschaden nach sich ziehen. Ein arterieller Mitteldruck unter $65 \mathrm{mmHg}$ sollte im Rahmen der Notfallversorgung nicht akzeptiert werden und muss behandelt werden. Eine Ausnahme kann eine permissive Hypotonie bei unstillbaren Blutungen als Teil des Behandlungskonzepts sein.

\section{Relevanz der koronaren Durchblutung}

Als „Motor“ des Kreislaufs benötigt das Herz Energie. In Ruhe sind ca. 10-11 ml Sauerstoff pro $100 \mathrm{~g}$ Gewebe pro Minute notwendig. Dieser Bedarf kann bei Anstrengung um das 4- bis 5-Fache erhöht sein [8]. Da der Patient 


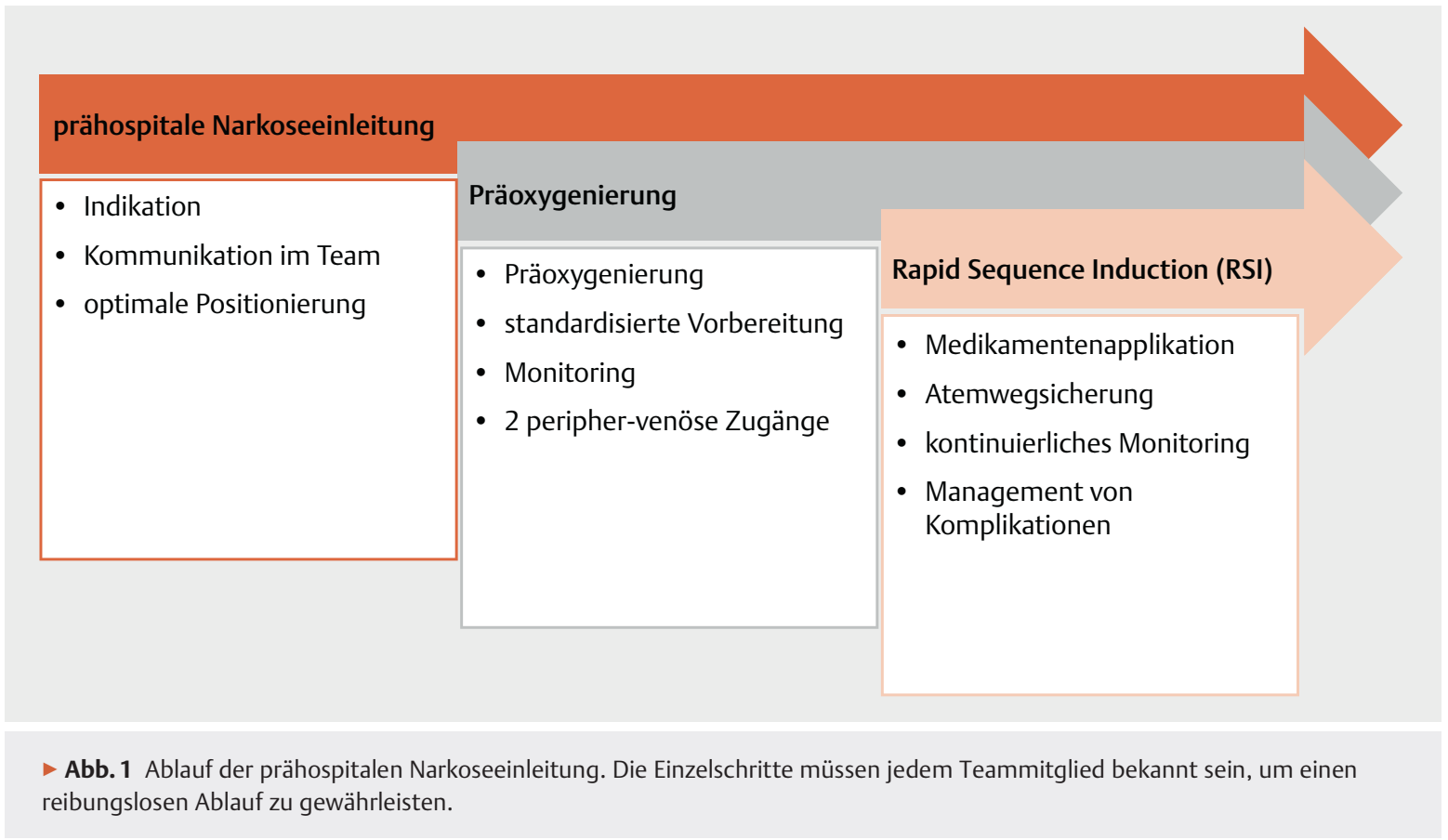

selbst bei gesundem Herzen in einer kritischen Notfallsituation maximal unter Stress steht, ist von einem erhöhten Bedarf auszugehen. Die Sauerstoffversorgung ist vom Perfusionsdruck an der Koronararterie abhängig, der sich aus dem enddiastolischen Druck minus des linksventrikulären enddiastolischen Drucks (LVED; ca. $6-12 \mathrm{mmHg}$ ) ergibt. Unterhalb von $50 \mathrm{mmHg}$ ist die Autoregulation des Koronargefäßes gestört [9], sodass die Interventionsgrenzen bei $60 \mathrm{mmHg}$ diastolischem Druck liegen sollten. Dieser gewinnt zunehmende Bedeutung, wenn die Koronarreserve durch Stenosen etc. gestört ist, wovon bei Patienten mit kardialer Indikation zur Narkoseeinleitung auszugehen ist.

\section{Nebenwirkung der Überdruckbeatmung}

Im Gegensatz zur Spontanatmung ist die treibende Kraft für den Luftstrom in die Lunge bei der maschinellen Beatmung ein positiver (Beatmungs-)Druck. Dadurch steigt der intrathorakale Druck im Vergleich zur Spontanatmung an und ist über den gesamten Atemzyklus im positiven Bereich. Der höhere intrathorakale Druck wird auf die großen venösen Gefäße übertragen und vermindert den venösen Rückfluss zum Herzen (Vorlast). Liegt die Indikation zur Narkose und Beatmung bei einer Lungenstauung mit Lungenödem, kann dies zu einer Optimierung des Verhältnisses von Herzfüllung und Schlagkraft (FrankStarling-Mechanismus) führen. Befindet sich der Patient allerdings infolge eines Volumenmangels bereits im Schock, führt der abnehmende venöse Rückstrom zur weiteren Eskalation.
Merke

Es sollten möglichst niedrige Spitzendrücke für die Beatmung genutzt werden. Spezielle Indikationen mit notwendigerweise höheren PEEP-Werten (z. B. Thoraxtrauma oder ARDS im Intensivtransport) stellen hier Ausnahmen dar.

\section{Nicht technische Fähigkeiten}

Der Rettungsdienst zählt zu den Hochrisiko-Organisationen [10]. Das Training nicht technischer Fähigkeiten (Aufgabenmanagement, Teamarbeit, Situationsbewusstsein, Entscheidungsfindung) [11] reduziert in diesen Organisationen Fehler und muss im Rahmen der Narkoseeinleitung beachtet werden [12].

\section{Ablauf der Narkoseeinleitung}

\section{Vorbereitung}

Trifft der Notarzt die Entscheidung zur Narkoseeinleitung, ist dies unverzüglich im Team zu kommunizieren. Der Gesamtablauf muss allen im Team bekannt sein ( $\triangleright$ Abb. 1). Jede Minute der Materialvorbereitung wird zur Präoxygenierung genutzt. Aus anderen Notfallsituationen mit Ad-hoc-Teams ist bekannt, dass die ruhige professionelle Ausstrahlung des Teams dem Patienten helfen kann [13]. Der Notfallsanitäter (NotSan) des Notarzteinsatzfahrzeugs (NEF) bereitet die Medikamente und die Intubation vor. Die Anlage von 2 peripheren Venenverweilkanülen ist obligat. Der Rettungssanitäter kann Monitoring und Absaugpumpe vorbereiten. Eine 


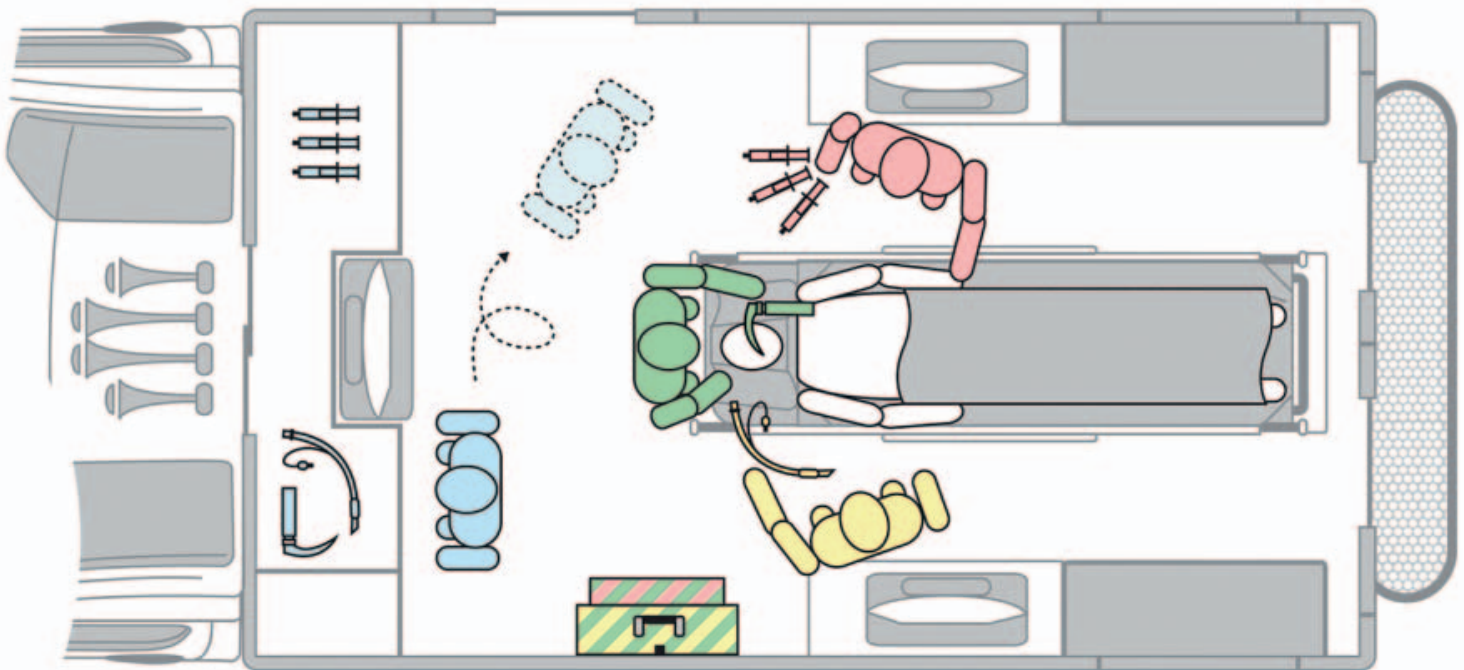

- Abb.2 Mögliche Raumaufteilung im Rettungswagen. Der Notarzt positioniert sich am Kopf des Patienten. Während der NEFNotSan die Medikation vorbereitet, steht der RTW-NotSan in Blickachse zum Monitor. Der Rettungssanitäter reicht Absaugkatheter und Intubation.

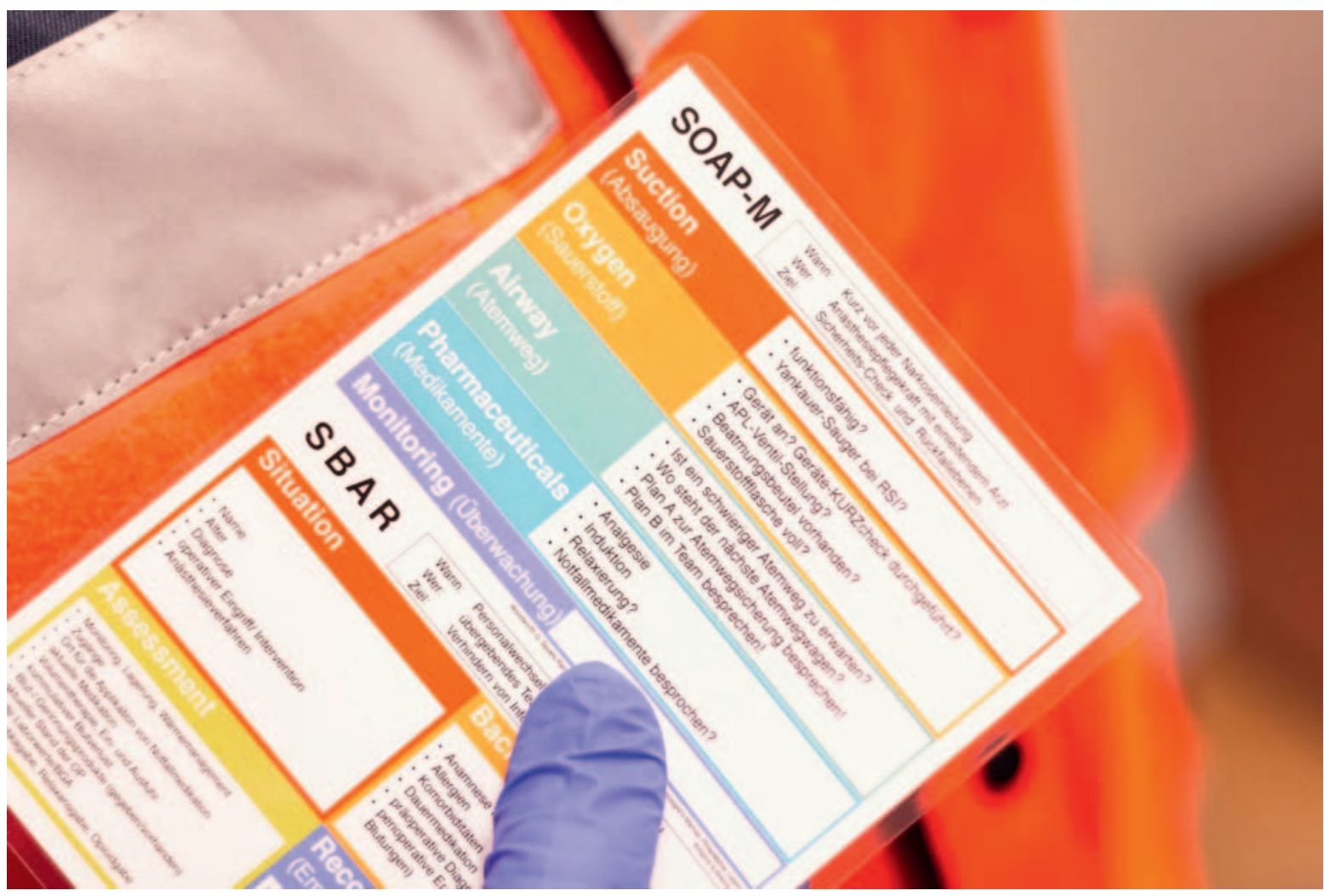

- Abb.3 Die SOAP-M-Taschenkarte ermöglicht kurz vor Narkoseeinleitung den letzten Check und erhöht die Durchführungssicherheit. $\mathrm{S}=$ Suction (Funktion, Absaugkatheter), $\mathrm{O}=$ Oxygen (Präoxygenierung), $\mathrm{A}=$ Airway (Atemweg, Plan B und $\mathrm{C}$ ), $\mathrm{P}=\mathrm{Pharma-}$ ka, $\mathrm{M}=$ Monitoring (sind alle Messwerte vorhanden und aktuell?).

mögliche räumliche Positionierung der Einsatzkräfte im Rettungswagen (RTW) zeigt $>$ Abb. 2.
Ein Kurzcheck nach dem SOAP-M-Schema hat sich vor der 1. Medikamentenapplikation („point of no return“) in unserem Arbeitsumfeld als hilfreich herausgestellt ( $\downarrow$ Abb. $\mathbf{3}$ ). 


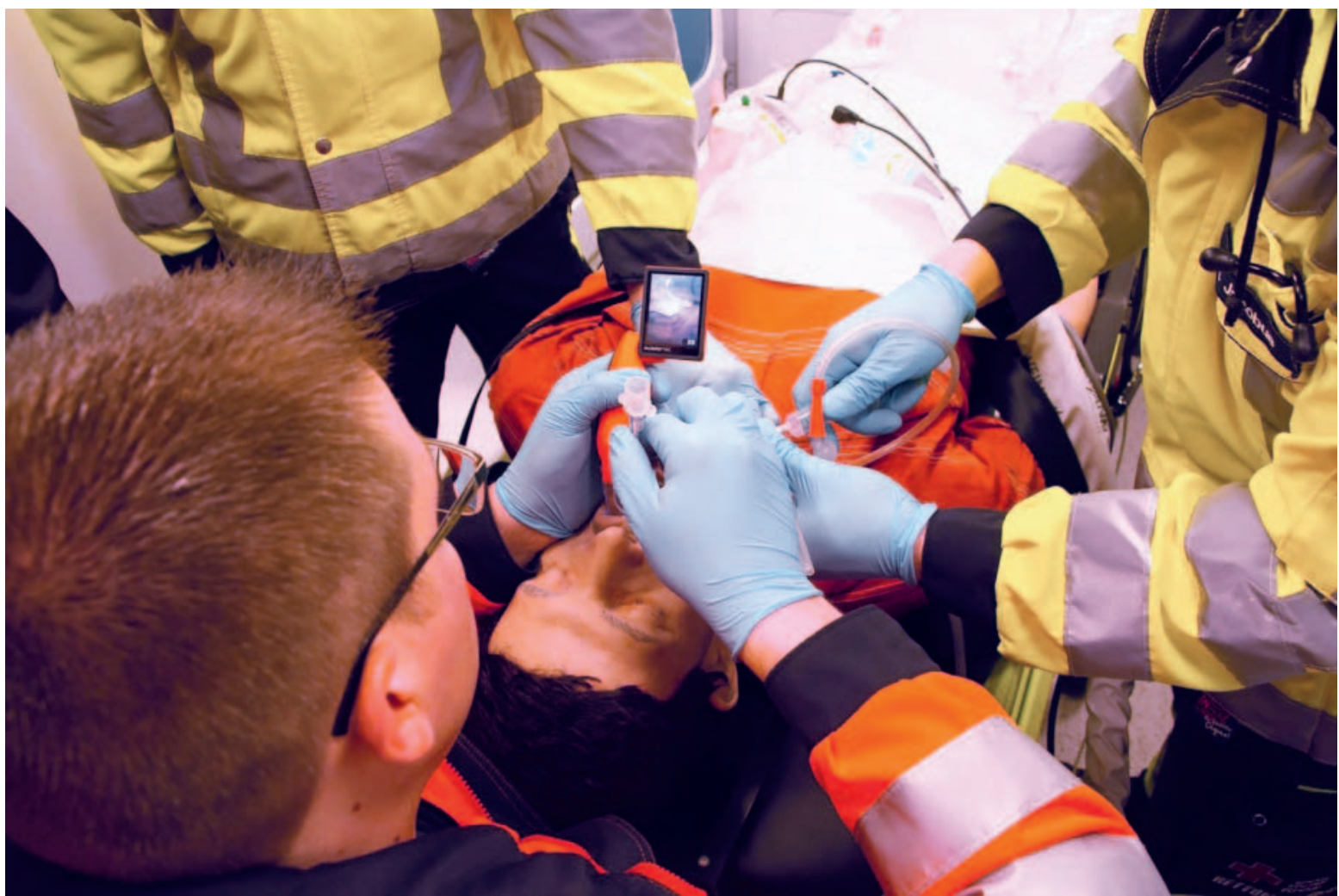

Abb.4 Blickwinkel des Notarztes. Der Notarzt hat aufgrund seiner Position am Kopf des Patienten einen eingeschränkten Blick auf vorbereitete Materialien oder das Vitaldaten-Monitoring. Eine gute Kommunikation im Team ist daher ein wichtiger Schritt zur optimalen Patientenversorgung.

Im Rahmen des Situationsbewusstseins werden Indikation und Verdachtsdiagnose reevaluiert und die erwarteten Schwierigkeiten kommuniziert.

\section{Durchführung}

Nachdem alle Teammitglieder mit dem Beginn der Narkoseeinleitung einverstanden sind, legt der Notarzt die Medikamentendosierungen fest. Im Rahmen einer geschlossenen Kommunikation werden alle Dosierungsangaben wiederholt und die definitive Applikation bestätigt. Die Pharmaka werden typischerweise in der Reihenfolge Opiat, Hypnotikum und Muskelrelaxans verabreicht.

\section{Merke}

Im Gegensatz zu früheren Meinungen (Opiat nach Intubation) führt die Opiatverwendung zu besseren Intubationsbedingungen und spart die Dosis an kreislaufwirksamen Hypnotika.

Ein zu erwartender starker Blutdruckabfall sollte vorausschauend mit einem Vasopressor behandelt werden. Erst bei ausreichendem Wirkeintritt der Medikamente (vor allem des Relaxans) wird die Atemwegssicherung durchgeführt. Die Blickachse des Notarztes erlaubt es während der Intubation nicht, gleichzeitig auf die Vitalfunktionen des Patienten zu achten ( $\triangleright$ Abb. 4). Dem Notarzt muss der Tubus in die Hand gegeben werden.

Dem NotSan in Blickachse des Monitors kommt während der gesamten Zeit der Einleitung die Aufgabe zu, die Vitalwerte des Patienten auf dem Monitor zu beachten und eine rechtzeitige Information über pathologische Entwicklungen in Hinblick auf die Zielwerte zu kommunizieren. Eine rechtzeitige Information und Intervention kann eine weitere Zustandsverschlechterung des Patienten verhindern.

Nach erfolgreicher Intubation und Lagekontrolle empfiehlt sich eine sofortige Beurteilung des Kreislaufs und der $\mathrm{CO}_{2}$-Ableitung.

\section{Aufrechterhaltung}

Nach Einleitung der Narkose und erfolgreicher Atemwegssicherung gilt es, einen zügigen Transport anzustreben. Das gewählte Hypnotikum für die Aufrechterhaltung der Narkose muss festgelegt und in Abhängigkeit von Ausstattung und Transportstrecke über einen Perfusor oder als Einzelboli gegeben werden. Bei der Übergabe an die behandelnden Krankenhausteams ist ein möglicher schwieriger Atemweg zu kommunizieren. Dies kann im 
Rahmen einer geplanten Extubation helfen, die durchführenden Teams entsprechend vorzubereiten.

\section{KERNAUSSAGEN}

- Die Narkoseeinleitung ist eine medizinisch komplexe Prozedur, die weit über die Maßnahme und Atemwegssicherung durch beispielsweise eine Intubation hinausgeht. Die erfolgreiche Behandlung erfordert ein interprofessionell gut agierendes Team.

- Auswirkungen der Nebenwirkungen bedürfen einer rechtzeitigen Intervention und vorausschauenden Arbeitens.

- Die Medikamentendosierung erfordert eine individuelle Anpassung an den jeweiligen Patienten (u. a. Köpergewicht, Kreislaufsituation). Unterschiedliche Halbwertzeiten müssen zwingend beachtet werden.

- Zum Erhalt der Hirnperfusion und der kardialen Funktion ist bei der Narkoseeinleitung ein Aufmerksamkeitsfokus auf systolischen, mittleren arteriellen Druck und diastolischen Druck wichtig.

- Es sollten möglichst niedrige Spitzendrücke für die Beatmung genutzt werden. Spezielle Indikationen mit notwendigerweise höheren PEEPWerten (z. B. Thoraxtrauma oder ARDS im Intensivtransport) stellen hier Ausnahmen dar.

- Der Kurzcheck SOAP ermöglicht die Überprüfung aller kritischen Materialien kurz vor dem „point of no return“.

- Im komplexen Geschehen darf nicht vergessen werden, dass der Verbleib am Einsatzort möglichst kurz gehalten werden muss, um den Patienten rechtzeitig einer klinischen Behandlung zuzuführen.

\section{Interessenkonflikt}

Markus Flentje und Hendrik Eismann haben 2018 und 2019 Referentenhonorare für Simulationstrainings mit dem Schwerpunkt prähospitale Narkose für Notärzte und Notfallsanitäter erhalten.

\section{Autorinnen/Autoren}

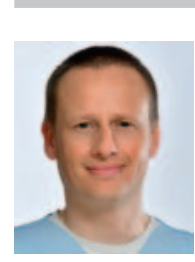

\section{Markus Flentje}

Dr. med. Markus Flentje DESA, Jahrgang 1976, Facharzt für Anästhesiologie. Tätig als Oberarzt an der der Klinik für Anästhesie und Intensivmedizin der Medizinischen Hochschule Hannover für den Bereich Aus-, Fort-, Weiterbildung. Interessensschwerpunkte: Training von NonTechnical-Skills, Curriculumentwicklung für Notfallteams.

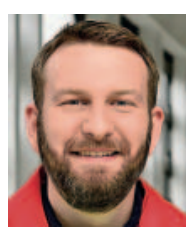

\section{Hendrik Eismann}

Dr. med. Hendrik Eismann, Jahrgang 1982, Facharzt an der Klinik für Anästhesiologie und Intensivmedizin der Medizinischen Hochschule Hannover. Leitender Notarzt. Interessenschwerpunkte: Teamtraining, „Shared Mental Models“, „Human Factors“ in der Medizin, Forschungsschwerpunkte: POC-Gerinnungsdiagnostik, „Cognitive Aids“ in der Notfallmedizin.

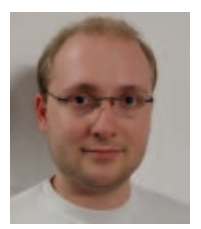

\section{Hendrik Fiedel}

Jahrgang 1991. Notfallsanitäter und Praxisanleiter bei der DRK Rettungsdienst in der Region Hannover gGmbH. Interessensschwerpunkte: Implementierung neuer Behandlungskonzepte in die Praxis, Ausbildungseffektivität in der Notfallsanitäterausbildung.

\section{Korrespondenzadresse}

\section{Dr. med. Markus Flentje}

Klinik für Anästhesiologie und Intensivmedizin Medizinische Hochschule Hannover

Carl-Neuberg-Straße 1

30625 Hannover

\section{Literatur}

[1] Bernhard M, Böttiger B, Bohn A et al. Handlungsempfehlung Prähospitale Notfallnarkose Erwachsene. Im Internet (Stand: 10.03.2020): https://www.awmf.org/uploads/tx_szleitlinien/ 001-030l_S1_Praehospitale_Notfallnarkose_Erwachsene_201503-verlaengert.pdf

[2] Timmermann A, Böttiger B, Byhahn C et al. Leitlinie Prähospitales Atemwegsmanagement. Im Internet (Stand: 10.03.2020): https://www.awmf.org/uploads/tx_szleitlinien/001040I_S1_Praehospitales-Atemwegsmanagement_2019-03_1. pdf

[3] Cook T, Behringer EC, Benger ]. Airway management outside the operating room: hazardous and incompletely studied. Curr Op Anaesth 2012; 25: 461-469

[4] Badjatia N, Carney N, Crocco T] et al. Guidelines for prehospital management of traumatic brain injury. 2nd ed. Brain Trauma Foundation, BTF Center for Guidelines Management. Prehosp Emerg Care 2008; 12: 1-52

[5] Willingham MD, Karren E, Shanks AM et al. Concurrence of Intraoperative Hypotension, Low Minimum Alveolar Concentration, and Low Bispectral Index Is Associated with Postoperative Death. Anesthesiology 2015; 123: 775-785

[6] Kertai MD, White WD, Gan TJ. Cumulative duration of „triple low" state of low blood pressure, low bispectral index, and low minimum alveolar concentration of volatile anesthesia is not associated with increased mortality. Anesthesiology 2014; 121 : $18-28$

[7] Striebel HW. Die Anästhesie. Stuttgart: Thieme; 2013

[8] Klinke R, Pape HC, Kurtz A, Silbernagl S. Physiologie. Stuttgart: Thieme; 2009

[9] Butterworth JF, Wasnick JD, Mackey DC. Morgan and Mikhail's Clinical Anesthesiology. 6th ed New York: McGraw-Hill Education/Medical; 2018 
[10] Hagemann V, Kluge A, Kehren C. Evaluation of Crew Resource Management: Interventions for Doctors-on-call 2015. Im Internet (Stand: 10.03.2020): http://hfes-europe.org

[11] Flin R, Glavin R, Maran N et al. Framework for Observing and Rating Anaesthetists' Non-Technical Skills - Anaesthetists' Non-Technical Skills (ANTS) System Handbook v1.0. 2012. Im Internet (Stand: 11.04.2020): https://www.abdn.ac.uk/iprc/ documents/ANTS\%20Handbook\%202012.pdf

[12] Schmutz ], Manser T. Do team processes really have an effect on clinical performance? A systematic literature review. $\mathrm{Br}$ J Anaesth 2013; 110: 529-544
[13] Flentje M, von Kaisenberg C, Achenbach J et al. Betreuung der Patientin während eines Notfallkaiserschnittes - Evaluation von Qualitätsmerkmalen aus Patientensicht. Z Geburtshilfe Neonatol 2018; 2: 1-10

Bibliografie

DOI https://doi.org/10.1055/a-0962-1802

retten 2020; 9: 190-198

(c) Georg Thieme Verlag KG, Stuttgart · New York

ISSN 2193-2387 


\section{Punkte sammeln auf CMEthieme.de}

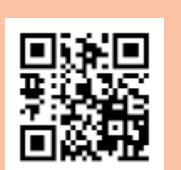

Diese Fortbildungseinheit ist in der Regel 12 Monate online für die Teilnahme verfügbar.

Den genauen Einsendeschluss finden Sie unter https://eref.thieme.de/CXDGUEA.

Sollten Sie Fragen zur Online-Teilnahme haben, finden Sie unter https://cme.thieme.de/hilfe

eine ausführliche Anleitung. Wir wünschen viel Erfolg beim Beantworten

der Fragen!

Unter https://eref.thieme.de/CXDGUEA oder über den QR-Code kommen Sie direkt zur Startseite des Wissenstests.

\section{Frage 1}

Welche Aussage zur Indikation der Durchführung einer prähospitalen Narkose trifft zu?

A Wenn absehbar ist, dass ein Patient im Verlauf operiert werden muss, sollte die Narkose möglichst zügig eingeleitet werden.

B Nach dem Einleiten der Narkose muss unverzüglich eine Hypoglykämie ausgeschlossen werden.

C Die prähospitale Narkoseeinleitung kann der Sicherstellung der Oxygenierung dienen.

D Die prähospitale Narkoseeinleitung ist aufgrund der vorgehaltenen übersichtlicheren Materialien komplikationsfreier als die intrahospitale Narkoseeinleitung.

E Findet die Narkoseeinleitung im Rahmen einer Reanimation statt, sind die Medikamente unverzüglich nach Eintreffen zu applizieren.

\section{Frage 2}

Welche Aussage zu den Rahmenbedingungen der prähospitalen Narkoseeinleitung ist richtig?

A Da alle prähospitalen Patienten als nicht nüchtern gelten, ist die Narkoseeinleitung immer als „rapid sequence induction“ durchzuführen.

B Die prähospitale Narkoseeinleitung ist aufgrund der optimalen Ausbildung aller Teammitglieder nahezu risikofrei.

C Aufgrund des hohen Handlungsdrucks reicht eine Pulsoxymetrie als Monitoring beim Patienten aus (Anzeigen von Oxygenierung und Perfusion).

D Ein EKG ist allen anderen Monitormaßnahmen vorzuziehen, da es die Kreislauffunktion am sichersten darstellt.

E Nach Applikation der Medikamente sollte im Rahmen eines SOAP-Sicherheitschecks das Material zur Intubation überprüft werden.

\section{Frage 3}

Welche Aussage zur Narkosetiefe und Applikation von Medikamenten trifft auf die prähospitale Narkoseeinleitung zu?

A Aufgrund der längsten Wirkdauer der Muskelrelaxanzien sind diese als erstes zu applizieren.

B Um Nebenwirkungen der Narkoseeinleitung zu reduzieren, sollten zur Einleitung keine Katecholamine und Vasopressoren gegeben werden.

C Die Nachinjektion von Narkosemedikamenten kann erfolgen, wenn der Patient eine klare Aufwachreaktion zeigt.

D Die unterschiedlichen Halbwertszeiten der Medikamente haben das Risiko einer nicht bemerkbaren Wachheit des Patienten zur Folge.

E Die Narkosemedikamente können problemlos in hohen Mengen appliziert werden, da ein Aufwachen des Patienten im RTW nicht gewünscht ist.

\section{Frage 4}

Welche Aussage zur Hirnperfusion bei der Narkoseeinleitung ist richtig?

A Ein systolischer Blutdruck von über $80 \mathrm{mmHg}$ sichert eine Oxygenierung des Gehirns.

B Der Patient sollte die doppelte Narkosemitteldosis wie üblich erhalten, um einer Stressreaktion entgegenzuwirken.

C Aufgrund der Narkose ist der Sauerstoffverbrauch des Gehirns sehr gering und vernachlässigbar.

D Wurde der Patient mit Kreislauf ins Krankenhaus gebracht, ist eine ausreichende Hirnperfusion während des Transports vorhanden gewesen.

E Um eine Minderperfusion zu vermeiden, sollte ein MAD von $<65 \mathrm{mmHg}$ nicht unbehandelt akzeptiert werden.

- Weitere Fragen auf der folgenden Seite... 


\section{Frage 5}

Welche Aussage zur Koronardurchblutung ist korrekt?

A Die Durchblutung der Koronarien findet während der Systole des Herzens statt, daher ist auf einen ausreichend hohen systolischen Blutdruck zu achten.

B Ist die Koronardurchblutung gefährdet, kann über eine Tachykardie (z. B. mittels Atropingabe) die Sauerstoffversorgung des Myokards verbessert werden.

C Die koronare Autoregulation sichert die Myokarddurchblutung auch in kritischen Phasen bei Blutdrücken unter $80 / 40 \mathrm{mmHg}$.

D Die koronare Durchblutung ist vom linksventrikulären enddiastolischen Druck und dem diastolischen Druck abhängig.

E Der Perfusionsdruck in den Koronarien sollte während der Narkoseeinleitung $15 \mathrm{mmHg}$ nicht unterschreiten.

\section{Frage 6}

Welche Auswirkung hat die maschinelle Beatmung auf den Patienten?

A Im Gegensatz zur Spontanatmung treten intrathorakal insbesondere in der Einatmung negative Drücke im Thorax auf.

B Durch die Verminderung der Vorlast kann der kardiale Output des Patienten sowohl steigen als auch fallen.

C Beim Patienten im Volumenmangelschock führt der hohe intrathorakale Druck zu einer Verbesserung des venösen Rückstroms.

D Um die Oxygenierung des Patienten sicherzustellen, muss durchgängig ein hoher PEEP eingesetzt werden.

E Die Anwendung eines möglichst hohen Beatmungsdrucks sichert ein ausreichendes Atemzugvolumen.

\section{Frage 7}

Was trifft auf die Vorbereitung der Narkoseeinleitung zu?

A Wenn der GCS des Patienten $<8$ ist, kann der Notarzt erwarten, dass das Rettungsteam bereits die Narkose vorbereitet.

B Der Notfallsanitäter des NEF soll bei der Vorbereitung zu Ausbildungszwecken dem Rettungssanitäter die Medikation erklären.

C Eine Aufgabenzuordnung im Vorfeld der Narkoseeinleitung ist sinnlos, da alle Teammitglieder alle Aufgaben erfüllen können.

D Das RTW-Team bereitet bestenfalls die gewünschten Medikamente aus dem Notarztkoffer vor.

E Die Vorbereitungszeit soll für eine Präoxygenierung des Patienten genutzt werden.

\section{Frage 8}

Welche Aussage zum Teammanagement bei der Narkoseeinleitung ist richtig?

A Das Checkprotokoll SOAP-M soll möglichst kurz vor der technischen Durchführung der Intubation durchgeführt werden.

B Das Checkprotokoll SOAP-M ermöglicht bei Dienstübernahme die Kontrolle, ob alle notwendigen Materialien im Notarztwagen vorhanden sind.

C Die professionelle Ausstrahlung des Teams wird dadurch unterstützt, dass die Teammitglieder möglichst wenig über die Indikation zur Narkose sprechen.

D Der „point of no return“ beschreibt den Zeitpunkt, zu dem klar ist, dass der Patient ins Krankenhaus transportiert wird.

E Die Narkose kann abhängig von der Transportstrecke mittels Einzelboli oder Dauerapplikation (Perfusor) von Medikamenten aufrechterhalten werden.

\section{Frage 9}

Welche Aussage zum Aufgabenmanagement bei der Narkoseeinleitung ist korrekt?

A Ein fachkundiges Teammitglied muss die Vitalwerte des Patienten im Blick behalten und im Verlauf bewerten.

B Der Notarzt muss als verantwortliche Person den Monitor und die Vitalwerte des Patienten ständig beobachten.

C Der Notfallsanitäter sollte die Intubation durchführen, damit er seine Handlungskompetenz aufrechterhält.

D Alle Teammitglieder positionieren sich vor den Schränken im RTW, um möglichst schnell benötigtes Material aus diesen anzureichen.

E Vor der Narkoseeinleitung sollte möglichst wenig kommuniziert werden, damit sich jedes Teammitglied voll auf seine Aufgabe konzentrieren kann.

\section{Frage 10}

Welche Aussage zum Patientenmanagement nach der Atemwegssicherung ist richtig?

A Sedierende Medikamente sollten im Verlauf des Transports nicht weitergegeben werden, da sie die klinische Untersuchungsmöglichkeit verhindern.

B Prähospital etablierte Intubationen gelten als einfach und bedürfen keiner speziellen Dokumentation.

C Die Durchführung als „rapid sequence induction“ erlaubt es dem Notarzt, ohne Hypnotikum zu intubieren.

D Nach der Narkoseeinleitung ist der Handlungsdruck in der Patientenversorgung vorbei, da die Vitalfunktionen des Patienten gesichert sind.

E Nach der Intubation ist unverzüglich eine Lagekontrolle des Tubus durchzuführen und die Kreislauffunktion zu überprüfen. 